\title{
Architecture Design for Unmanned Aerial Vehicle Mission Planning System
}

\author{
Xiaopeng $\mathrm{Ji}^{*}$ and Yanjie Zhao \\ No.11 Shuangyuan Road, Shijingshan District, Beijing, China \\ ${ }^{*}$ Corresponding author
}

\begin{abstract}
Keywords: Unmanned aerial vehicle (UAV), Mission planning system (MPS), Data management, Resource allocation.
\end{abstract}

\begin{abstract}
Unmanned aerial vehicle (UAV) mission planning system (MPS) refers to assigning missions to each UAV according to the missions to be accomplished, the number of UAVs and the different mission payloads, and making flight routes through route planning technology. In this paper, starting from the concept of UAV mission planning, the information requirement environment and construction requirement of UAV mission planning system is analyzed. Then the basic architecture and modular design of UAV mission planning is summarized. At last, the problems of UAV mission planning and the development trend of UAV mission planning system is discussed.
\end{abstract}

\section{Concept of UAV Mission Planning}

With the continuous progress of modern information technology and the development of information warfare, UAV's combat mission has developed from single mission to multi-mission. It has developed from target-based to reconnaissance and surveillance missions. At the same time, it has developed to target location and fire negotiation, communication relay, electronic warfare, ground attack, air early warning and other directions. The tactical application of UAVs has also developed from single combat to formation operations between UAVs and hybrid formation operations between UAVs and manned fixed-wing aircraft/armed helicopters. The development of UAV combat mission and the change of tactical application put forward new requirements for mission planning. The requirement of intelligence, effectiveness and real-time decision-making is much higher than before. However, the traditional manual/semi-manual method for UAV mission planning has a long response time and weak processing capacity, which cannot fully utilize the performance of UAV, and it is difficult to meet the operational requirements.

UAV mission planning system (MPS) is based on the missions to be accomplished, the number of UAVs and the different mission payloads, assigning missions to each UAV and formulating flight routes through route planning technology. In the process of flight, UAV mission payloads can be controlled in order to complete reconnaissance, attack, evaluation and other missions. The main functions of UAV mission planning are as follows:

(1) Mission allocation [1, 2]: Through the mission planning system, reasonable plan is carried out among multi-mission, multi-objective and multi-UAV. The different payloads and performance of UAV is fully considered and the UAV and its payload resources are reasonably allocated. The objective is to minimize the time and cost to achieve and complete the assigned missions.

(2) Route planning [3, 4]: Formulate the flight path of the take-off, landing, approaching to the monitoring point, reconnaissance of monitoring area, departure from the monitoring point, return flight and emergency flight of the aircraft, which requires that the threat cost of missiles, radar, etc. and fuel consumption cost of the UAV are minimized.

\section{Process of UAV Mission Planning}

The main objective of UAV mission planning is to plan one or more optimal or satisfactory routes for UAV from the starting point to the target point based on terrain information and enemy 
information. The performance, arrival time, fuel consumption, threat and flight area of UAV are taken into account, so as to ensure UAV to complete its mission successfully and return to base safely. In the structure of command and control system, mission planning is often expressed as a node. Figure 1 illustrates the information flow shared among mission planning systems, navigation, payload information analysis and databases, and illustrates the various types of data required in mission planning and their relationships.

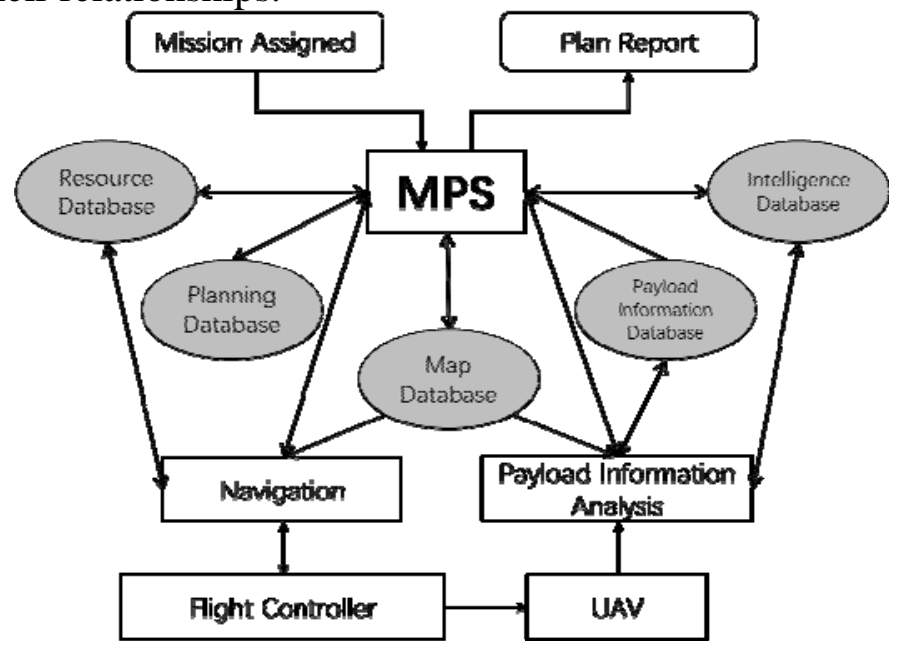

Figure 1. Information flow in MPS environment

\section{System Process}

Mission planning of UAV is essentially a map-based process, which begins with mission delivery and ends with planning one or more paths to meet mission requirements and other criteria. Mission requirements usually refer to UAV observing a place or area through airborne sensors and payloads, analyzing and returning relevant data within a specified period of time. Other standards include collision avoidance, threat avoidance, fuel consumption control, compliance with air traffic control rules and so on. Generally, there is a trade-off between these criteria. For example, achieving all the mission goals may require too much fuel so that less important targets can be removed from the plan.

During mission execution, the navigation unit receives UAV status data, such as position, heading, fuel volume and ground speed. These related data are also stored in the resource database and monitored by MPS during mission execution.

For some reason, additional mission objectives which are not included in the original plan may be required. At this time, the ground controller can apply for the guidance unit to temporarily take over the control of the UAV and control it remotely. In order to facilitate the successful completion of the mission, mission planning system needs to analyze the submission strategy and put forward the corresponding constraints, that is, where the UAV can fly, how long it takes, whether the fuel range and survival probability are satisfied. Then the ground controller abandons the remote control of the UAV, and the UAV restores the original path plan.

The specific planning results obtained by the system include resource allocation of combat aircraft, target allocation of enemy and flight path. In the process of mission execution, all units and command headquarters in the combat network can access the information database remotely or receive telemetry information directly from UAV to obtain timely and effective reference information.

\section{Database}

Resources, planning, maps, results and intelligence databases are used to store information needed to assist UAV planning and management missions to meet operational requirements. The contents of each database are as follows:

Resource Database: The basic database which stores battlefield operation parameters and the status of each equipment unit, including UAV, target, payload, transmitter, antenna, etc. Battlefield parameters define the boundary of equipment performance. The UAV status data includes flight 
parameters and fuel volume, which are received directly from the UAV during the mission. Payloads include different sensor accessories, transmitters and antennas. Resource database can provide radar modeling data and UAV feature models from all angles to help UAV fly in a certain attitude to reduce the risk of detection by enemy sensors.

Planning Database: The database which stores past, present (ongoing) and new (forthcoming) mission plans.

Map Database: The database which stores electronic maps of various sizes, including digital terrain elevation data, terrain features, vegetation, river systems and topographic maps of artificial terrain. Map data comes from different sources and needs to be processed in different formats. The updating period of map data is long so it is necessary to acquire more detailed topographic data with satellites.

Payload Information Database: The database which stores information acquired by previous and current UAV missions, mainly images. Images from different sources and institutions may have different formats, so images from other UAVs and satellites need to be processed and stored in this database.

Intelligence Database: The database which stores intelligence assessment, surface to air missile (SAM) location report, electromagnetic interference area which hinders UAV communication, weather area which is unfavorable to flight and reduces sensor image quality, frequency channel, battle area boundary and rules of engagement, intersection location, safe route and airspace control rules at different time of day, etc.

Objects stored in different databases need to be cross-referenced. For example, an image evaluation stored in an intelligence database may involve multiple images in the image database, while the relevant geographic reference map data is located in the map database.

\section{Functional Modules of UAV Mission Planning System}

Mission resource allocation and available path generation after mission assignment are completed by mission planning system. Mission resource allocation includes target allocation, payloads allocation, take-off location and sorties batch determination. The specific composition of MPS depends on the planning process mentioned above. As shown in Figure 2, the common functional modules include:

Data Management Module: Building basic database, including resources, planning, results, maps and information database. It has the functions of importing, exporting, editing, checking and retrieving data based on query.

Resource Allocation Module: Including payloads allocation, target partition, aircraft batch, equipment satisfaction calculation and configuration results output. It mainly completes the determination of combination between UAV and target, payload, reasonable division of target area, determination of take-off and landing location of aircraft and other functions.

Route Calculation Module: Including the generation of initial route, route adjustment and smoothing, and route verification. Simply put, under the constraints of enemy threat and terrain threat, an optimization algorithm is used to calculate the safe, effective and near-optimal flight path between the aircraft and the target. According to the aircraft's dynamic characteristics, weather and electromagnetic interference, the flight path is adjusted and smoothed from time to altitude. Then the flight path is checked to judge the effectiveness of the mission.

Distribution Module: Interconnecting with each unit in the combat network, submitting and distributing reports after completing mission planning and mission completion.

Simulation Evaluation Module: The two-dimensional and three-dimensional simulation of the mission planning results can visually display the process of UAV mission execution and then evaluate it. 


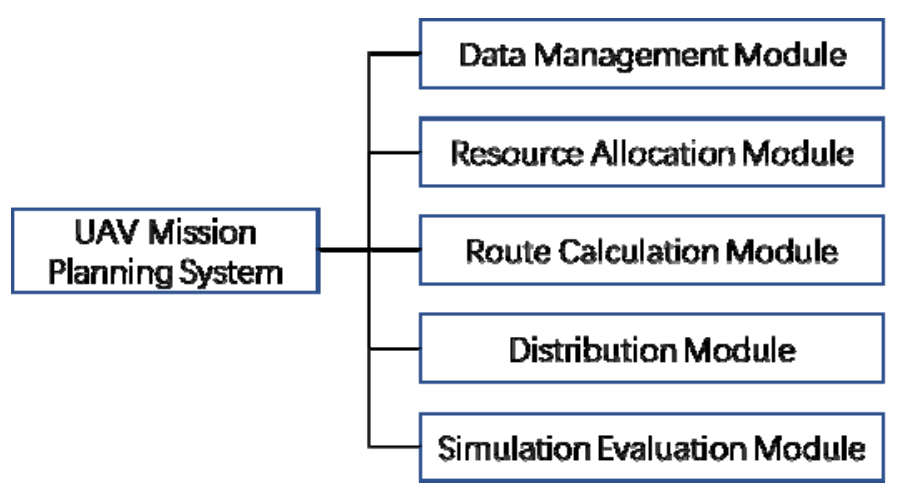

Figure 2. Structure diagram of UAV mission planning system

It should be pointed out that a mission planning system does not need to be precisely divided according to the above components, but it must meet the requirements involved in the above items. Each module needs to share the resource database, payload information database, intelligence database, map database and planning database.

\section{Data Management Module}

The efficiency of mission planning system basically depends on the accuracy, timeliness and completeness of data in database. The system needs all kinds of data. The data sent to the system must be received, recorded and processed in an appropriate format before it can be stored in the relevant database. Operators need to be able to create and modify mission plan based on information decisions. The general requirements of data management module include:

(1) Mission planning system must be able to run whether map information is available or not. In particular, MPS must be able to scan survey tables and geographic references, which are stored in map databases. It must also be able to process various coordinate types of data, but the data format must conform to the national or military information format standards.

(2) Information related to the location of enemy forces and threat areas must be used to update the information in the intelligence database. Missions must specify the list of target locations and the names and coordinates required for each target.

(3) Each mission must specify the time limit for the information of each target required by the mission unit. These limitations include the time to reach the target, the ideal cruise time, and the completion of the cruise no later than a certain point in time. For long-term missions, these times must also specify different time zones.

(4) Equipment must be able to receive digital maps from other sources and store them in map databases for supplementing and updating topographic maps.

(5) Data can be readily accepted during mission execution so that mission plans can be based on up-to-date information. Mission information must be entered into the planning database, and they will also be cross-referenced by their corresponding missions.

\section{Resource Allocation Module}

Resource allocation module adopts certain operational principles and allocation algorithms to achieve efficient and reasonable allocation of all kinds of resources needed for action, such as aircraft, payload, target, etc. The goal of resource allocation is to maximize the benefits of these actions, to minimize resource consumption, or to combine the two. The main contents of resource allocation include equipment allocation, mission target partition, batch and sortie calculation, equipment satisfaction calculation, etc. Equipment configuration mainly refers to the choice of payload for UAV. According to the reconnaissance requirements of the mission, the weather, threat information and enemy target information in the mission execution area are analyzed, and the load type and quantity are determined. Mission target partition is based on the type of reconnaissance payload and target and its geographical location, using classification algorithm to classify the target. Then according to the geographical distribution and following the principle of proximity for spatial clustering, the target area is reasonably divided. Batch and sorties are calculated by weighting the 
comprehensive factors of route length and aircraft survival probability. The calculation of equipment satisfaction degree is the final requirements to overall calculate whether the takeoff airport can meet the requirements of various types of equipment.

\section{Trends and Prospects}

In the future, mission planning system will develop towards online real-time planning, integrated mission and route planning, etc. The main performances are as follows:

(1) Real-time online planning. After mission pre-allocation, with the continuous change of battlefield situation, it is necessary to constantly adjust and modify the original mission and route. When new missions are changed or added, the new route network for mission execution can be quickly planned from the perspective of the overall situation of the fleet. When sudden threats occur or the original threat prediction is faulty, the route for avoiding threats can be planned in real time. Since the UAV is flying at a high speed in the combat process, it is impossible to make detailed evasion of all the routes that need to be updated every time when new missions or sudden threats are encountered. Planning requires a compromise between real-time and optimality. The mission can be quickly decided by the route prediction method, and a series of better predicted routes can be quickly provided to ensure that the mission assignment can be completed in a relatively short time. Therefore, the algorithm is required to have high efficiency and meet the real-time requirements.

(2) Integrated planning. At present, the technical research is relatively simplified, and each of them carries out one aspect of the research, without forming a unified function. For example, the research of mission planning system is mostly divided into many points, such as task allocation technology [1, 2], route planning technology [3, 4], collision avoidance control technology [5-7], etc.

(3) Cooperative operational planning. Current research focuses on multi-objective task scheduling, load allocation and route optimization of single UAV. In order to improve UAV combat effectiveness, future UAV cluster operations, UAV cooperative networking operations and multiple UAVs are required to accomplish missions together. Therefore, the planned route is required to ensure that UAVs reach the target airspace at the same time in order to coordinate operations.

(4) Mission payload and function are more detailed. In domestic and foreign research, most task allocation models focus on multi-aircraft single task single target, multi-aircraft single task multiobjective, single-aircraft single task multi-objective and single-aircraft multi-task multi-objective. There is little research on multi-aircraft multi-task multi-objective. Some multi-aircraft multi-task multi-objective research is also limited to multi-aircraft multi-task allocation of different targets, without considering that the same combat mission requires multi-sorties of UAVs.

(5) Mission re-planning. Current research is limited to one-time task scheduling in static battlefield, and no secondary task scheduling is carried out according to battlefield damage assessment after an attack.

\section{Conclusion}

The mission planning system of UAV is a complex integrated system, which allocates resources and plans route before and during the flight of UAV. When the combat mission or battlefield environment changes, UAV should be able to complete the mission in real time through system adjustment. However, the functional requirements of mission planning system largely depend on the actual mission requirements and resource allocation capabilities, and there is no fixed pattern. Therefore, the future research focus of UAV command and control mission planning system should be oriented to the direction of modularization, versatility and portability. In this paper, the architecture of mission planning system and the design of related modules are summarized based on the development of practical application, which can provide relevant reference for the practical application of UAV. 


\section{References}

[1] Alighanbari M. Task assignment algorithms for teams of UAVs in dynamic environments[D]. Massachusetts Institute of Technology, 2004.

[2] Forsmo E J, Grøtli, E.I, Fossen T I, et al. Optimal search mission with Unmanned Aerial Vehicles using Mixed Integer Linear Programming[C]// International Conference on Unmanned Aircraft Systems. IEEE, 2013.

[3] O'Rourke K P, Carlton W B, Bailey T G, et al. Dynamic Routing of Unmanned Aerial Vehicles Using Reactive Tabu Search[J]. Military Operations Research, 2001, 6(1):5-30.

[4] Wu J, Yi J, Gao L, et al. Cooperative path planning of multiple UAVs based on PH curves and harmony search algorithm[C]// Computer Supported Cooperative Work in Design (CSCWD), 2017 IEEE 21st International Conference on. IEEE, 2017.

[5] Yu T, Tang J, Bai L, et al. Collision avoidance for cooperative UAVs with rolling optimization algorithm based on predictive state space[J]. Applied Sciences, 2017, 7(4): 329.

[6] Besada-Portas E, de la Torre L, Jesus M, et al. Evolutionary trajectory planner for multiple UAVs in realistic scenarios[J]. IEEE Transactions on Robotics, 2010, 26(4): 619-634.

[7] Sujit P B, Beard R. Multiple UAV path planning using anytime algorithms[C]// 2009 American Control Conference. IEEE, 2009: 2978-2983. 